Modern Physics Letters A

(C) World Scientific Publishing Company

\title{
THE PROTON-DEUTERON BREAK-UP PROCESS IN A THREE-DIMENSIONAL APPROACH
}

\author{
IMAM FACHRUDDIN* \\ Institut für Theoretische Physik II, Ruhr-Universität Bochum, 44780 Bochum, Germany \\ CHARLOTTE ELSTER \\ Institut für Kernphysik, Forschungszentrum Jülich, 52425 Jülich, Germany \\ Institute of Nuclear and Particle Physics, Ohio University, Athens, OH 45701, USA \\ WALTER GLÖCKLE \\ Institut für Theoretische Physik II, Ruhr-Universität Bochum, 44780 Bochum, Germany \\ Received (received date) \\ Revised (revised date)
}

\begin{abstract}
The pd break-up amplitude in the Faddeev scheme is calculated by employing a threedimensional method without partial wave decomposition (PWD). In a first step and in view of higher energies only the leading term is evaluated and this for the process $\mathrm{d}(\mathrm{p}, \mathrm{n}) \mathrm{pp}$. A comparison with the results based on PWD reveals discrepancies in the cross section around $200 \mathrm{MeV}$. This indicates the onset of a limitation of the partial wave scheme. Also, around $200 \mathrm{MeV}$ relativistic effects are clearly visible and the use of relativistic kinematics shifts the cross section peak to where the experimental peak is located. The theoretical peak height, however, is wrong and calls first of all for the inclusion of rescattering terms, which are shown to be important in a nonrelativistic full Faddeev calculation in PWD.
\end{abstract}

\section{Introduction}

A three-dimensional (3D) approach without partial wave decomposition (PWD) has been developed for the nucleon-nucleon $(\mathrm{NN})$ system, which uses directly the relative momentum vectors, together with a helicity representation of the total spin. It has been successfully applied to NN scattering ${ }^{1}$ and the deuteron ${ }^{2}$, using the realistic NN potentials Bonn- $\mathrm{B}^{3}$ and AV18. ${ }^{4}$

We extend this approach to the three-nucleon $(3 \mathrm{~N})$ break-up process. In a first step we consider only the leading term in the multiple scattering series, which is in first order in the NN t-matrix $T$. The interest is twofold: we want to see the onset of the limitations of a standard PWD by going to higher energies and whether this first order treatment is sufficient. In addition, the shifts in the observables by changing from nonrelativistic to relativistic kinematics will be investigated.

*Permanent address: Jurusan Fisika, Universitas Indonesia, Depok 16424, Indonesia 
The Proton-Deuteron Break-Up process in a Three-Dimensional Approach

\section{Formulation}

The leading term of the pd break-up amplitude in the Faddeev scheme is given by

$$
U_{0}=\left\langle\mathbf{p q} m_{1} m_{2} m_{3} \tau_{1} \tau_{2} \tau_{3}|(1+P) T P| \phi\right\rangle=U_{0}^{(1)}+U_{0}^{(2)}+U_{0}^{(3)},
$$

where $\mathbf{p}, \mathbf{q}$ are Jacobi momenta, $m_{i}, \tau_{i}(i=1,2,3)$ spin and isospin quantum numbers, $T$ the NN t-matrix, $P=P_{12} P_{23}+P_{13} P_{23}$ permutation operators, $|\phi\rangle=$ $\left|\mathbf{q}_{0} m_{1}^{0} \tau_{1}^{0}\right\rangle\left|\varphi_{d} M_{d} \tau_{d}\right\rangle$ the initial state and $U_{0}^{(i)}(i=1,2,3)$ the three parts of the amplitude resulting from $(1+P)$.

Now $U_{0}$ can be expressed in terms of the t-matrix $T_{\Lambda \Lambda^{\prime}}^{\pi S t}\left(q, q^{\prime}, \cos \theta, E\right)$ in the momentum-helicity basis, for given parity, total spin and isospin, and final and initial helicities $\Lambda^{\prime}$ and $\Lambda .{ }^{1}$ Since $U_{0}^{(2)}, U_{0}^{(3)}$ are related to $U_{0}^{(1)}$ by means of permutations, it is sufficient to work out only $U_{0}^{(1)}$. We obtain

$$
\begin{aligned}
U_{0}^{(1)}(\mathbf{p}, \mathbf{q})= & (-)^{\frac{1}{2}+\tau_{1}} \delta_{\tau_{2}+\tau_{3}, \tau_{1}^{0}-\tau_{1}} \frac{1}{4 \sqrt{2}} \sum_{m^{\prime}} \sum_{l=0,2} Y_{l, M_{d}-m^{\prime}-m_{1}}\left(\hat{\pi}^{\prime}\right) \varphi_{l}\left(\pi^{\prime}\right) \\
& C\left(\frac{1}{2} \frac{1}{2} 1 ; m_{1} m^{\prime}, m^{\prime}+m_{1}\right) C\left(l 11 ; M_{d}-m_{1}-m^{\prime}, m_{1}+m^{\prime}\right) \\
& \sum_{S \pi t}\left(1-\eta_{\pi}(-)^{S+t}\right) C\left(\frac{1}{2} \frac{1}{2} t ; \tau_{2} \tau_{3}\right) C\left(\frac{1}{2} \frac{1}{2} t ; \tau_{1}^{0},-\tau_{1}\right) e^{-i\left(\Lambda_{0} \phi-\Lambda_{0}^{\prime} \phi_{\pi}\right)} \\
& C\left(\frac{1}{2} \frac{1}{2} S ; m_{2} m_{3} \Lambda_{0}\right) C\left(\frac{1}{2} \frac{1}{2} S ; m_{1}^{0} m^{\prime} \Lambda_{0}^{\prime}\right) \sum_{\Lambda \Lambda^{\prime}} d_{\Lambda_{0} \Lambda}^{S}(\theta) d_{\Lambda_{0}^{\prime} \Lambda^{\prime}}^{S}\left(\theta_{\pi}\right) \\
& e^{i\left(\Lambda^{\prime} \phi^{\prime \prime}-\Lambda \Omega\right)} T_{\Lambda \Lambda^{\prime}}^{\pi S t}\left(p, \pi, \cos \theta^{\prime \prime} ; E_{d}+\frac{3}{4 m}\left(q_{0}^{2}-q^{2}\right)\right)
\end{aligned}
$$

with $\boldsymbol{\pi} \equiv \frac{1}{2} \mathbf{q}+\mathbf{q}_{0}$ and $\boldsymbol{\pi}^{\prime} \equiv-\mathbf{q}-\frac{1}{2} \mathbf{q}_{0}$. Besides standard notations there occur the deuteron wave function components $\varphi_{l}\left(\pi^{\prime}\right) . U_{0}^{(2)}$ and $U_{0}^{(3)}$ are obtained by suitable replacements of the momenta and discrete quantum numbers.

\section{Results and Discussions}

Here we use the NN potentials Bonn-B and AV18. Figs.1(a) and (b) compare our $3 \mathrm{D}$ results to the ones based on the PWD, both first oder in $T$, for the cross section and the analysing power $A_{y}$ in the process $d(p, n) p p$. The PWD calculation includes the NN t-matrix for total NN angular momenta $j=5$ and 7 , and states of total $3 \mathrm{~N}$ angular momenta up to $J=31 / 2$. We see a significant discrepancy in the peak of the cross section but agreement for $A_{y}$ in case of $j=7$. Since $j=7$ is not feasible right now in a full Faddeev calculation (all orders in $T$ ) one has apparently reached the limits of a generally reliable PWD at such an energy of about $200 \mathrm{MeV}$. At lower energies, say $100 \mathrm{MeV}$, the PWD agrees perfectly well with our 3D results. In Figs.1(c) and (d) we show the full Faddeev results for the cross section and $A_{y}$, now based on $j=5$ in the PWD. The figurues reveal that rescattering is quite important, even at this relatively high energy.

In Fig. 2 we compare our calculated cross section to data ${ }^{5}$. The $3 \mathrm{D}$ result (called "3D nonrel") clearly deviates by giving the peak at the wrong position. Next we use 
The Proton-Deuteron Break-Up process in a Three-Dimensional Approach
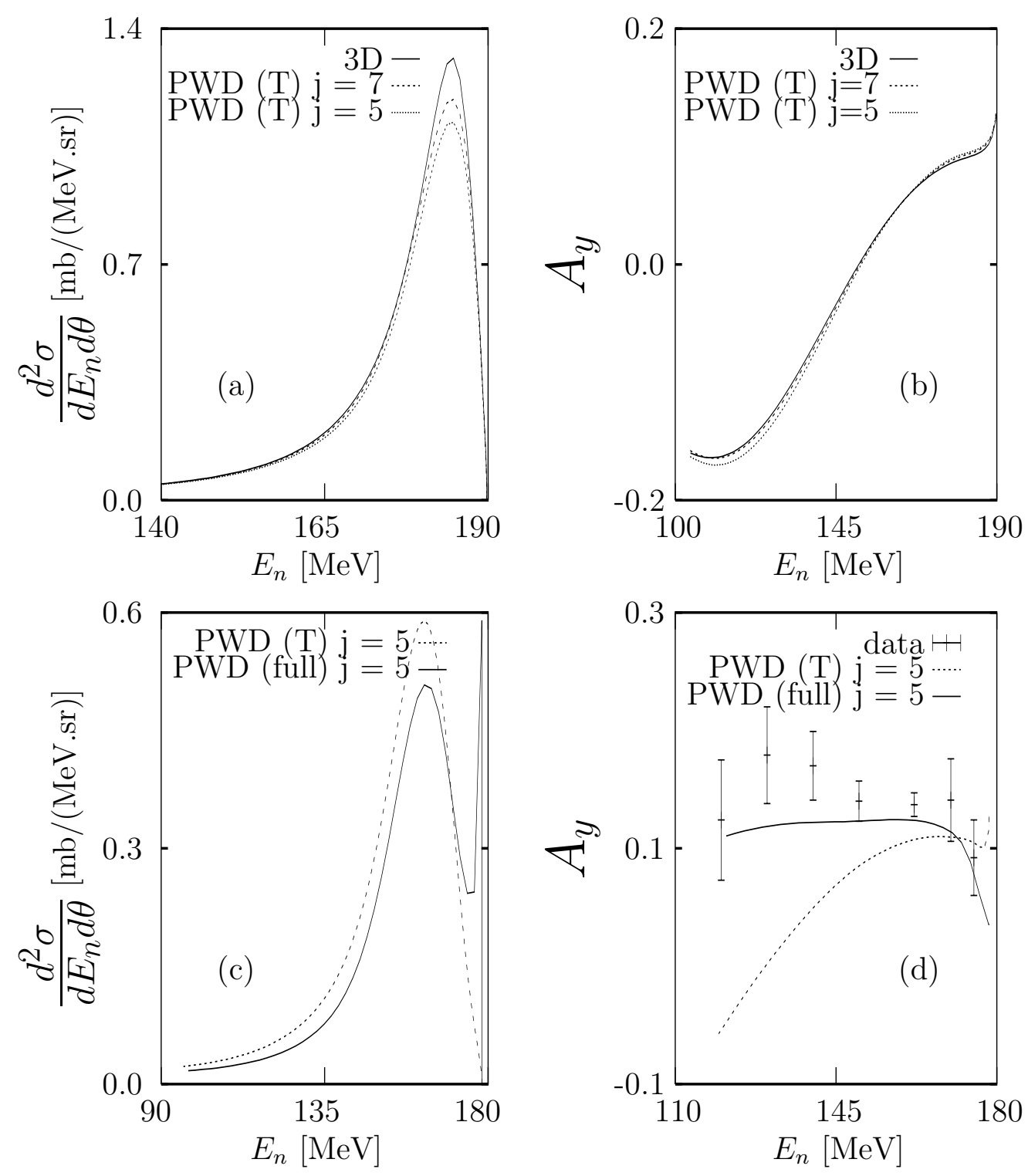

Figure 1: The spin averaged differential cross section (a \& c) and the analyzing power $A_{y}(\mathrm{~b} \& \mathrm{~d})$ at $E_{l a b}=197 \mathrm{MeV}, \theta=13^{0}(\mathrm{a} \& \mathrm{~b})$ and $\theta=24^{0}(\mathrm{c} \& \mathrm{~d})$ for the process $d(p, n) p p$.

relativistic kinematics by employing appropriately Lorentz boosted momenta ${ }^{6}$. This shifts the curve "3D nonrel" to the one labeled "3D rel", which gives the correct peak position. However, this calculation overshoots the data. Thus, our calculations of the break-up cross section indicate that one needs altogether three ingredients: 


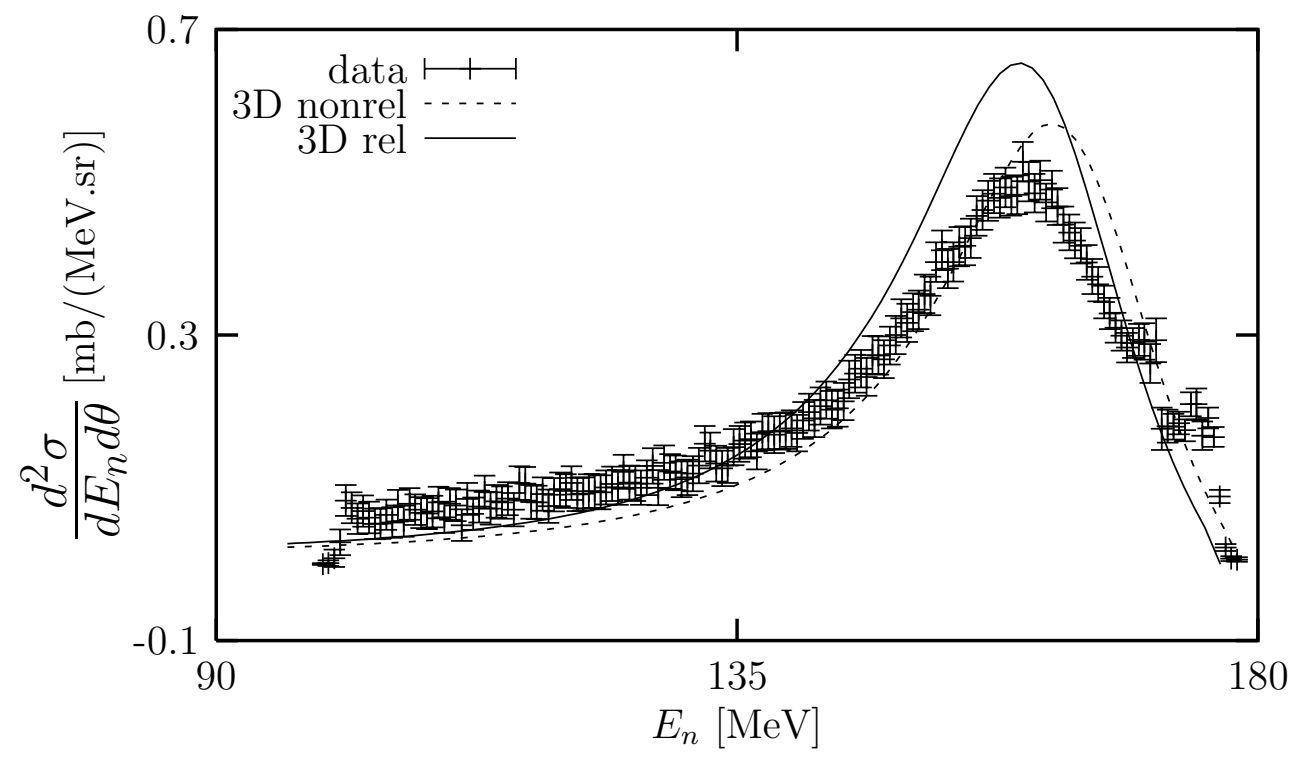

Figure 2: The spin averaged differential cross section for the process $d(p, n) p p$ at $E_{l a b}=197 \mathrm{MeV}, \theta=24^{0}$.

a 3D treatment, rescattering effects and relativity. For $A_{y}$ shown in Fig.1(d) the full nonrelativistic Faddeev calculation in the PWD is closed to the data. Since the studied relativistic effects for $A_{y}$ are quite small (not shown) and the higher partial waves appear unimportant as also shown in Fig.1(b) that agreement will presumably survive. In case of the cross section we see in Fig.1(c) a decrease due to rescattering, which might cure the overshooting seen in the relativistic curve of first order in $T$ in Fig.2. More details, especially on various spin observables in the process $d(\vec{p}, \vec{n}) p p$ at $197 \mathrm{MeV}$ and higher energies can be found $i^{7}$ and in a forthcoming publication.

\section{Acknowledgments}

We would like to thank H. Witala and J. Golak from Instytut Fizyki, Uniwersytet Jagiellonski, Cracow, Poland for providing the PWD calculations.

\section{References}

1. I. Fachruddin, Ch. Elster, W. Glöckle, Phys. Rev. C62, 044002 (2000).

2. I. Fachruddin, Ch. Elster, W. Glöckle, Phys. Rev. C63, 054003 (2001).

3. R. Machleidt, Adv. Nucl. Phys. 19, 189 (1989)

4. R. B. Wiringa, V. G. J. Stoks, and R. Schiavilla, Phys. Rev. C51, 38 (1995).

5. D. L. Prout, et.al., Phys. Rev. C65, 034611 (2002).

6. R. Fong and J. Sucher, J. Math. Phys. 5, 456 (1964).

7. I. Fachruddin, PhD. thesis, Ruhr-Universität Bochum, 2002. 